\title{
Cognitive Activity: Philosophical Analysis, Psychological and Pedagogical Characteristics
}

\author{
Ekaterina Korobova $^{1}$, Irina Kardovich $^{1}$, Marina Konysheva $^{2 *}$ and Dina Mironova ${ }^{1}$ \\ ${ }^{1}$ Plekhanov Russian University of Economics, Moscow, Russian Federation \\ ${ }^{2}$ Russian University of Transport (RUT-MIIT), Institute of Legal Studies, Moscow, Russian Federation
}

\begin{abstract}
Cognition is an integral part of a person's orientation in the world. It is studied by different sciences - philosophy, sociology, pedagogics, and psychology. In information society, the role of cognition and the ways it is realized are becoming extremely important. Cognitive activity is an essential part of cognition. It is formed and developed in the process of education. Cognitive activity is traditionally regarded as a special kind of mental activity. There are different components of cognitive activity and levels of its development. The higher the level of cognitive activity is, the more efficient the whole process of education is as knowledge is acquired more quickly and at a higher level. Cognitive independence combined with cognitive activity can radically improve the whole process of cognition. Thus, the task of an educational process is to activate cognition and develop cognitive independence by different methods and approaches.
\end{abstract}

\section{Introduction}

Cognition is a socio-historical process of human activity aimed at reflecting objective reality in a person's mind. Modern philosophy defines cognition as a creative activity, orientated towards obtaining reliable knowledge about the world. Cognition is also seen as an essential characteristic of culture. [1, p. 527]. In the modern society, where science and technology are becoming an increasingly important determining factor of social development, the role of scientific knowledge is constantly growing. It does not only opens up unlimited prospects for the civilization development, but also, solves the problem of choosing the pathway, for civilization, determines its prospects, defines its goals, develops the means and methods for achieving them [2, p. 5].

Cognition is an integral part of a person's existence in the world. Cognition is studied by many special sciences that explore its various aspects and problems philosophy, sociology, pedagogy, psychology.

The theory of cognition is a fairly widely developed theme in philosophy. But, despite this, the topic has not lost its relevance in our time, on the contrary, it seems expedient to consider the issues related to a person's cognitive activity today, in connection with the formation of new economic relations that have led not only to the changes in the material sphere, but also to the global changes in the spiritual sphere. Today, the problem of a person's creative self-development and self-education, their adaptation to the changing conditions of life is vital. The possibility of such development arises due to cognitive activity and mental independence, which open a wide field of activity in the sphere of education and self-education [3, p. 482].

\section{Methodology and research methods}

The research was based on the method of comparative analysis of cognition and cognitive activity from the point of view of different sciences, of cognitive activity formation and mental independence in the process of education.

\section{Results and discussion}

Among many needs that ensure the socio-cultural life of people, the need to cognize is one of the earliest and most essential for their existence. This need received its theoretical justification in a number of philosophical and special scientific disciplines, as a result of which the doctrine of cognition was developed (the theory of knowledge).

Philosophy considers cognition as a complex process of perceiving the essence of objects including both the external analysis of the phenomenon and the analysis of its essence, starting from the study of a particular object to the study of the system of interrelations between objects. Cognitive activity of a person is based on their sensory, material activity. In the process of development, our knowledge is becoming more specific, and objective. The criteria and the only means of verifying the truth of our knowledge is practice. Practical activity itself is always based on certain knowledge and theoretical assumptions. People act consciously, guided by their 
existing ideas about reality, setting themselves certain specific goals. In the unity of practice and theory, a complete objective activity is carried out - the process of practical and theoretical development of the world by people. Practical activity reveals how correctly ideas and theories reflect the essence of the phenomena.

In the information society, the problem of obtaining and assimilating knowledge is one of the central for the development of the whole society. At the same time, the issues and character of the cognition theory are changing. There are new ways to discuss and solve traditional problems, and new issues and problems have been appearing that never existed in the classical theory of cognition [4].

The first stage of cognition begins with a living contemplation, perception, on the basis of which representations arise in the human brain. These primary representations, united and transformed by imagination, can form more complex representations, but they yield to knowledge realized through abstract thinking in their depth of penetration into the object. Nevertheless, abstract thinking is an indirect and generalized reflection in the human brain of what is given by living contemplation, is the cognition of the essence of properties, causal relationships and regular connections of things.

Cognition in the form of consciously creative, predictive and transforming activity is an exclusively human way of reflecting reality and necessarily supposes the activity and independence of the subject.

As we have mentioned above, cognition is studied by different sciences. From the point of pedagogy, the study of cognitive activity is essential, since the future of civilization depends on people, their ideas, ways of life, the whole complex of their behavioral attitudes. What moral values will people learn, how will they apply them in real life? Will the people direct their abilities, their intellect, their will for the good of the world, or remain an indifferent observer of current events - what kind of people they will be - depends on those to whom the great and noble mission of personal development is entrusted [5, p. 12], that is to teachers.

The object of pedagogical science is education, which is the transfer of the generations' experience, the involvement of human beings in the life of society, the assimilation and development of social experience by individuals [6, p. 6]. To identify, study, systematize and apply in practice the basic laws and principles of knowledge means to answer the main questions of pedagogy - how and what to teach. Moreover, in an industrial society with its time deficit and the growing information flow, the issue of how to optimize the learning process, how to help an individual to learn more within a shorter period, how to teach them not to be lost in the amount of information and facts that they receive is especially important.

In pedagogy, there are different views on cognition in general and on cognitive activity in particular. In modern pedagogical science, there are different paradigms of education: authoritarian and humanistic.

The first direction, authoritarian pedagogy, refers to the traditional educational paradigm of the objective type and comes from the authority of the teacher. Teaching according to this paradigm is aimed at transferring the ready-made conclusions of science (by a teacher, teaching tools), facts, patterns, principles and rules to the students, instead of teaching students to find them independently, i.e. to teach methods and ways of solving problems [7, p. 26]. With this paradigm of learning, the educational material is considered as a kind of didactically prepared scientific knowledge [8, p. 16]. Such a direction does not deny the student's role, but treats them as a passive listener and observer, the object of pedagogical influence, that should be encouraged to assimilate material, develop their abilities within the content of instruction [9]. The leading role is assigned to the teacher, who transfers knowledge to the student. In conventional practice, the vast majority of academic time is occupied by information-communicating methods and technologies [10, p. 27]. With authoritarian guidance, the teacher is as active as possible. This approach equips the student with a certain amount of knowledge relevant at the time of its acquisition, but does not teach to acquire new knowledge independently as the old one becomes obsolete or to apply knowledge in an unconventional situation when an original decision is required.

Humanistic pedagogy (an innovative paradigm of the subjective type, the development of which began in the second half of the nineteenth century) puts student in the center of learning process, it affirms the activity of student who acts as a responsible subject of independent cognitive activity aimed at satisfying socially significant basic needs of the individual. In this way learning acquires the person-oriented character. Humanistic pedagogy contributes to the disclosure of students' capabilities. Cognition is realized through joint efforts of both the student and the teacher. In return, the student enriches the teacher in the same way as the teacher helps the student to cognize the world. Teaching students, the teacher cognizes themselves, reveals the essence of man as such and thus rises to the heights of professionalism. The main task of Humanistic pedagogy is the student's self-realization. Such active role of the student in cognitive activity substantially optimizes the process of acquiring knowledge, improves its effectiveness. Without denying the need to acquire knowledge, this direction in pedagogy seeks to develop a creative personality capable of applying this knowledge in new conditions, to move away from the standard model when making decisions. The central place in the given pedagogical paradigm is the problem of formation of the learner's cognitive activity as a necessary condition for the development of the creative personality. The selfdestructive pedagogy of coercion is completely replaced by the pedagogy of cooperation, which establishes the student's connections with the outside world as a single system and orientates toward friendly relationship between the teacher and the student and leads to creative studying [11, p.41].

There is a large number of definitions of cognitive activity and various characteristics of its features and attributes. Cognitive activity is the degree of the student's energy in cognition ..., which is characterized by the student's participation in the search for the 
unknown [12, p.45]. Cognitive activity is defined as both a personality trait, and an individual's selective activity aimed at transforming the object and achieving the task and goal [13, p. 91], and as "the student's active state, which is characterized by the desire for learning, mental strain and the manifestation of willful efforts" [14, 57].

A common position in these definitions is that cognitive activity is characterized through cognitive work and efforts. Simultaneously, cognitive efforts are conditioned by the contradictions of the cognizing subject and the cognizable object as a source of personal development and transformation of the surrounding reality; the efforts have a selective, motivated, socially significant character, a creative direction, intellectual tension, creative independence. Cognitive efforts are a necessary condition for the birth and growth of cognitive activity, as well as its result.

In the Soviet psychology, a detailed theory of activity was developed by A.N. Leontiev. The activity structure includes the following components: the need - the motive - the goal - the conditions for achieving it (the unity of purpose and conditions determines the task) and the activities related to them - the action (the unit of activity - contains all its specific characteristics) operations. We can speak about a person's specific activity only when its needs and motives are highlighted with respect to any of their activities, with sufficiently clear characteristics of the content. The concept of activity must be connected with the concept of motive. The actions that are undertaken by people are the basic components of individual human activities. Action is the process specified by the idea of the result to be achieved, that is, the process aimed at achieving a particular goal. Just as the concept of motive is correlated with the concept of activity, the concept of goal is correlated with the concept of action [10, p. 215]. An essential component of the activity is the means and norms for its implementation. Objective characteristics of the activity include specific conditions for its implementation and its final results [10, p. 304].

M.S. Cagan believes that the essence of human activity is the transformation of reality, an active impact on it; activity is the basic form of the social nature of man [15, p. 32].

Activity is one of the most important attributes of human existence, connected with a purposeful change in the external world, the people themselves. It is through activity that the essence of man is revealed [1, p. 21]. In any activity, a person acts as an active bearer of their social essence. Any human activity consists of the following components: goal-setting, transforming, prediction.

Studies in the field of philosophy, sociology, psychology and pedagogy have shown that educational cognitive activity possesses all the basic features typical for any human activity. A comprehensive analysis of educational cognition has shown that the consistent implementation of the principle of activity in teaching is considered the main condition for the success of individual cognition in learning. Without active and purposeful cognitive and practical activities, it is impossible to master the academic disciplines effectively.

Activity as a phenomenon exists in animate and inanimate nature. As a philosophical category, activity is understood by most philosophers as a universal feature, an attribute of matter, but this feature is inherent only to living systems. Human activity is aimed at the formation of one's own personality, and this process takes place only in activities or, according to A.N. Leontiev, the internal subject acts through the external and thereby changes itself [10, p. 180].

T. I. Shamova identifies two meanings of the term "activity":

- component of any process of interaction, determined by the intrinsic nature of the object;

- a process whose nature is manifested primarily by the internal determination of the object, its selfconditioning, in this internal determination dominates over the external one [16, p. 15].

Cognitive activity and independence are characteristics of human cognitive actions and, at the same time, essential qualities of a person capable of cognizing the surrounding world in the process of its transformation. These qualities are defined as emotionalvolitional, moral and intellectual readiness of the subject to cognize. Typically, it is viewed as the tension of mental forces, the manifestation of initiative, cognitive interest, efforts in acquiring knowledge [17].

Activity is a way of interaction in the material world, the realization of opportunities, which causes complex and varied changes both in the surrounding reality, being the object of activity, and the personality as its subject. One of such changes is the emergence and development of cognitive activity as an integral feature of the human personality. Activity as a personal characteristic expresses a particular state of the person and their attitude to activity (attentiveness, inclination, active participation in the process, prompt response to changing circumstances of the activity). Personal activity is one of the central issues of a person's development [18, p. 25].

Cognitive activity is a component of general activity, a special kind of mental activity. This is a complex integral quality of the developing personality. It is different from simple training activities because with the latter, the student's work can be reduced to simple reproductive activities. However, cognitive activity is an independent student's activity at the productive, creative level.

Educational cognitive activity is shown by the following indicators:

- the selectivity of the approach to the objects of cognition,

- setting for oneself the goal, the task to be solved,

- the transformation of the object in the activity aimed at solving the problem;

- the desire to gain new knowledge;

- target perception;

- creative search of ways and means to solve the problem;

- $\quad$ application of these means to achieve the purpose; 
- tension, concentration and sustainability of attention;

- volitional efforts;

- mental activity;

- the desire to move away from the template;

- initiative [19, p.32];

- aspiration on their own motivation to participate in the activities;

- active application of acquired knowledge and skills;

- the desire to share new information with others;

- emotional attitude to the activity;

- willingness to act (concentration of attention, reaction in case of difficulties - desire to overcome them, desire to finalize training activities;

- free choice of activities - the choice of books, the choice of activities during leisure hours. [20, p.104-109];

- cognitive purposefulness, expressed in verbal and non-verbal activity of students, qualitative knowledge of the subject (high final score).

Studying cognitive activity, researchers also pay attention to whether the student is ready to actively master knowledge with persistent, systematic volitional efforts, the ability to self-study, plan independently, organize the cognition process, monitor and evaluate the obtained results.

The development of cognitive activity is directly connected with the formation of cognitive interest - the desire to cognize an object or phenomenon, to master one or another type of activity. Interest is selective and acts as one of the most significant incentives for acquiring knowledge [21, p. 373]. If there is a strong interest in mastering knowledge, the knowledge is acquired better; it becomes firm as interest is the person's perception of the subject as something of valuable and attractive.

Researchers distinguish such components of cognitive activity as intellectual (or cognitive), motivational, (or pragmatic), emotional [22].

In accordance with these components, the criteria for the formation of cognitive activity are stipulated:

- cognitive (academic achievement, knowledge quality);

- motivationally-demanding (presence of cognitive interest, involvement in extracurricular forms of work, etc.) [23];

- operational-practical (ability to solve creative tasks, readiness for independent work, etc.);

- emotional (the reaction to the content of the information received, to how the process of educational mental activity is organized).

Depending on how well cognitive activity is formed and developed, three levels of cognitive activity are usually distinguished: high, medium and low. Some scientists give their own definitions of the levels. G.I. Shchukina defines the levels of student's cognitive activity as follows:

1. Reproductive-imitative - the experience of an activity is gained through the experience of another person. At this level, the person's activity is insufficient.
2. Search-performing activity is characterized by greater independence and is a higher level of activity.

3. Creative activity - the highest level of activity. If such activity is formed, the person sets tasks, chooses new, unconventional ways of solving the problem. [24, p. 27].

E.V. Korotaeva suggests dividing the levels of cognitive activity depending on how they manifest themselves into:

1. Zero (passivity, expectation of habitual pressure from the teacher, refusal to solve the problem independently);

2. Relatively-active (interest in certain learning situations associated with emotional attraction);

3. Executive-active (the ability to subordinate emotional, intellectual and volitional efforts to specific learning goals);

4. Creative (typical for capable and gifted students) [25].

T.I. Shamova identifies reproducing, interpreting and creative cognitive activities [17, p. 196].

High (creative) cognitive activity is characterized by the desire for cognition, mental effort and the demonstration of learners' moral volitional qualities. Cognitive activity is of an individual character. Significant signs of high activity are a high intellectualindicative reaction to the content of the educational material on the basis of the emerging cognitive need, the implementation of a number of consistent and interrelated actions aimed at achieving a certain cognitive result.

The criterion for the formation of this level of cognitive activity can be the student's interest in understanding the phenomena and processes studied, independent search for a problem solution in the process of cognitive activity, cognitive consistency, competence, etc. A characteristic indicator of this level of cognitive activity is the demonstration of strong willed qualities of the learner, concentration, perseverance and persistence in achieving the goal, broad and stable cognitive interests.

The average (search-performing) level of cognitive activity displays itself in recurrent manifestations of the need for cognitive activity and the average level of proficiency. Students have both active and passive periods, a great dependence on the existing conditions, lack of initiative, sluggishness.

The low (reproductive-imitative) level of cognitive activity is characterized by no definite motives of activity, passivity in self-improvement, the lack of initiative and creativity, inability to put knowledge into practice and lack of desire to do it.

Consequently, when students achieve a high level of cognitive activity in the process of learning, the acquired knowledge will be of higher quality as a result. Since activity is one of the necessary conditions for cognition, then the person involved in the learning process should be as active as possible. The aim of the teacher is to contribute in every possible way to the development of cognitive activity of the students.

The basis for increasing cognitive activity is correlation between mental and practical activities. This 
principle of the unity of consciousness and activity was formulated by psychologist S.L. Rubinshtein. He stated that the real human consciousness can not be detached from the activity in which it is implemented and formed in the same way as motive-based and focused human activity can not be isolated from the psyche, from the consciousness [26, p. 192].

S.L. Rubinshtein came to the conclusion that learning presupposes certain internal conditions that ensure this process and lead to the creation of new internal conditions that are necessary for acquiring the subsequent knowledge.

This is achieved by the goal-oriented activity of the teacher, aimed at improving the content, forms, methods, means of education, in order to stimulate interest, increase activity, creativity, student mental independence, by more effective means, methods and forms of teaching that promote cognitive activity.

Many scientists associate the activation of cognition with so-called "problem-based teaching" (when the teacher organizes relatively independent search activity, during which students get new information), the use of information-communication technologies (ITC), the professional-oriented content of education (in higher education), the combination of classroom and extracurricular forms of work [27].

Some types of classical problem-based teaching have been developed: business and simulation games, process modeling (by means of computer as well), case analysis, brainstorming, heuristic discussion and others. In practice, such problem-based teaching has given the best results, in comparison with purely reproductive education, typical for authoritarian pedagogy.

Activation of cognition is possible through developing a system of methods and approaches, finding the most effective modes of training, the rational programming of practical situations corresponding to those conditions in which the application of professional knowledge and skills is necessary. The following components are the necessary conditions for the formation of cognitive activity:

- teaching should be based on active thinking of students;

- teaching should correspond to the optimal level of students development;

- a positive emotional tone in the learning process is necessary.

Thus, the teacher activates students' cognition. As a result of this effort, cognitive activity of students is gradually formed, which affects the whole process of cognition.

One of the most important components of cognitive activity development is the formation of cognitive independence.

Cognitive independence is associated with the cognitive activity of students in the learning process. Cognitive activity presupposes a certain level of independent ideas. The development of activity creates the necessary conditions for the development of independence. Only being constantly involved in the active study of the material, students begin to direct their actions; as a result, elements of cognitive independence arise in their activity. Independence, having arisen on the basis of activity, has a positive effect on the activity itself. As Z.I. Zagvyazinsky notes, “independent work contributes to deepening, expansion and systematization of knowledge, the development of motivation for cognition, forms the student's desire for constant replenishment and renewal of knowledge, teaches to use proper and rational timing in specific conditions" [28, p. $71]$.

It is cognitive independence combined with cognitive activity that can radically improve the whole process of cognition, since no object, no phenomenon of both material and abstract world, is formed in a person's mind without independent cognitive actions.

Independence means a set of different properties, for example, the ability prioritize, generalize, reveal the essence of phenomena, establish cause-effect relationships, curiosity, interest, and many others. Cognitive independence is not limited to the ability of the student to work independently under the supervision of the instructor. The key of cognitive independence is that it is invariant with respect to any activity, from educational to professional. S.L. Rubinshtein considers that the genuine independence assumes conscious motivation of actions and their validity. Freedom from other people's influences and opinions is not a willfulness, but an authentic manifestation of the independence of the will [29, p. 405]. Knowledge which is acquired by self-directed learning remains in memory for a longer period, and the search for information itself is an important cognitive process.

Higher education, following school, is a new stage in the life of a person on their way to the world of cognition, the stage of realizing their place in life. The key focus here should be on preparing the student for their future professional occupation [30]. Besides, vocational training in higher education cannot do without cognitive activity formation. It is necessary to encourage the students' activities not only by means of certain methods and approaches, but by inspiring the whole process and developing a system of methods and techniques that enhance the students' activity [31, p.39].

K.Sh. Akhiyarov and Yu.P. Pravdin conveyed the principle of cognitive activity formation in higher education in the following way, "cognitive activity of the student is an intensive learning process, conditioned by the need and readiness of the person for intellectual and professional activity, ensured by the optimal level of teaching and the availability of learning, resulting in the formation of a student as a subject of education" [27, p.56].

In higher education, the main emphasis should be made on forming a culture of students thinking (since in the era of scientific and technological progress, the information communicated to the student becomes obsolete earlier than a young specialist will have time to apply the received knowledge in practice), and not on transferring a certain amount of information to students. Higher education should encourage people to think, give impetus to their development and self-improvement

It is becoming increasingly urgent to teach a specialist to independently acquire knowledge and 
creatively use the information already available. With the current system of higher education, students receive about $40 \%$ of knowledge from the teacher, while the remaining $60 \%$ find by themselves. Moreover, this ratio is gradually changing in the direction of more independent work of students.

Teachers should include in their training process such aspects that would provide a dialectic of cognition, revealing the many-valued and multiple connections of phenomena and processes. The rational use of reproductive and productive components, the complexity of learning activities and their interrelations, the growing research and creative component in them ensures a smooth transition of students to scientific thinking. Research activity of students increases their independence and initiative in the process of cognition [32].

To ensure effective training of the future specialist, it is necessary to put the student in conditions close to their future occupation, which will help to critically shorten the period of adaptation of a young specialist, and enable them to apply knowledge more effectively for solving specific professional tasks.

\section{Conclusion}

The undertaken analysis leads to the conclusion that cognitive activity is an intensive learning process stipulated by the needs of the individual. Cognitive activity is not an inherent feature of the personality; it is formed in the process of cognition on condition of a high level of cognitive independence of the individual. A person's professional career and their efficiency as a specialist is by large determined by their cognitive activity at different levels of training period.

\section{References}

1. The newest philosophical dictionary (Minsk, 527, 21, 1999).

2. I.P. Farman, Theory of knowledge and the philosophy of culture (M., Nauka, 1986)

3. E. Malushko, O. Maletina, V. Lizunkov, V. Tsybaneva, International Multidisciplinary Scientific Conferences on Social Sciences and Arts, 481-487 (2016).

4. N.Yu. Fominyh, O.I. Barsova, M.V. Zarudnaya, N.V. Kolomijceva, Humanitarian education in an economic institution Materials In the International Scientific and Practical Correspondence Internet Conference 2, 366-378 (2017).

5. E. Yu. Malushko, International Multidisciplinary Scientific Conferences on Social Sciences and Arts, 1155-1160 (2015)

6. K.A. Shvartsman, Philosophy and upbringing (M., Nauka, 1998)

7. P.I. Pikasisty, Pedagogy (Moscow, 1998)

8. L.F. Machneva, Problems of linguistics and methods of teaching foreign languages IV, 26, (2000)

9. E.F. Zeer, G.M. Romantsev, Pedagogy 3, 16, (2002)
10. E. Malushko, O. Maletina, V. Tsybaneva, Advances in Social Science Education and Humanities Research 97, 175-180 (2017)

11. A.N. Leontiev, Activity, Consciousness. Personality, 27, 180, 215, 304, (1975)

12. Courier of UNESCO, A man playing, 41, (July 1977).

13. A.M. Matyushkin, Pedagogy 5, 45, (1978)

14. A.A. Balaev, Active methods of training (M., Profizdat, 1986)

15. I.F. Kharlamov, Pedagogy (M., Higher School, 1990)

16. M.S. Kagan, Human activity: The experience of system analysis (M., Politizdat, 1974).

17. T.I. Shamova, Activation of the teaching of schoolchildren (M., Ped-ka, 1982)

18. V.G. Lizunkov, V. I. Marchuk, E. Yu. Malushko, European Proceedings of Social and Behavioural Sciences 19, 456-463 (2017)

19. D.B. El'konin, Pedagogy 25, 104-109, (1986)

20. T.I. Shamova, Activation of the teaching of schoolchildren (M., Ped-ka, 1982)

21. Russian Pedagogical Encyclopedia, (M., 1993)

22. P.V. Sukhanov, Development of cognitive activity of future officers in a military high school, Author's abstract of the candidate of pedagogical sciences, (Orenburg, 2001)

23. A. Hassan, I. Al-Jubari, JSSH 24(3), Article ID: JSSH-1291-2015, (2016)

24. G.I. Shchukina, Activation of cognitive activity of students in the learning process (M., 1979)

25. I.B. Korotyaeva, Business game as a means of developing cognitive and professional interests of students, Author's abstract, dis. Cand. ped. Sciences, (Kiev, 1989).

26. S.L. Rubinshtein, Pedagogy 192, 405 (1973)

27. K. Meenakshi, JSSH 24 (4), Article ID: JSSH-13532015 (2016)

28. V.I. Zagvyazinsky, Didactics of the higher school (Chelyabinsk, 1990).

29. L.A. Vovsi-Tille, Professional education 10, 29-30, (2002)

30. R.A. Nizamov, Psihologo-pedagogical bases of development of cognitive activity of students (Sat. Kazan, Izd. Kaz. University, 1980)

31. K.Sh. Ahiyarov, Yu.P. Pravdin, Formation of cognitive activity of students in the process of education (Ufa: BGPI, 1988)

32. O.T. Ergunova, V.G. Lizunkov, E. Yu. Malushko, V. I. Marchuk, A. Yu. Ignatenko, IOP Conference Series-Materials Science and Engineering, 177 (2016) 